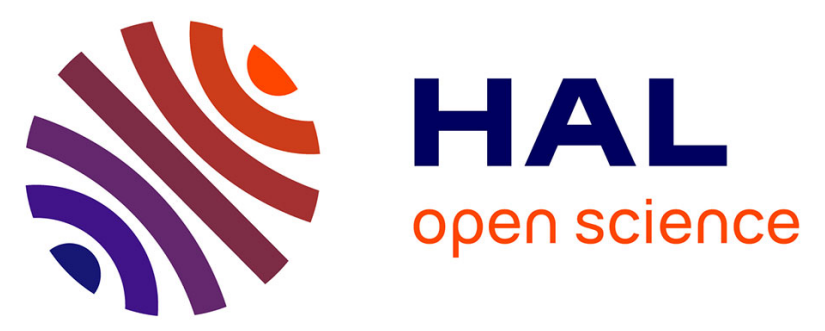

\title{
Widely Tunable Parametric Amplification and Pulse Train Generation by Heating a Photonic Crystal Fiber
}

Alexandre Kudlinski, Arnaud Mussot, R. Habert, T. Sylvestre

\section{To cite this version:}

Alexandre Kudlinski, Arnaud Mussot, R. Habert, T. Sylvestre. Widely Tunable Parametric Amplification and Pulse Train Generation by Heating a Photonic Crystal Fiber. IEEE Journal of Quantum Electronics, 2011, 47 (12), pp.1514-1518. 10.1109/JQE.2011.2173159 . hal-00655030

\section{HAL Id: hal-00655030 \\ https://hal.science/hal-00655030}

Submitted on 11 May 2021

HAL is a multi-disciplinary open access archive for the deposit and dissemination of scientific research documents, whether they are published or not. The documents may come from teaching and research institutions in France or abroad, or from public or private research centers.
L'archive ouverte pluridisciplinaire HAL, est destinée au dépôt et à la diffusion de documents scientifiques de niveau recherche, publiés ou non, émanant des établissements d'enseignement et de recherche français ou étrangers, des laboratoires publics ou privés. 


\title{
Widely Tunable Parametric Amplification and Pulse Train Generation by Heating a Photonic Crystal Fiber
}

\author{
Alexandre Kudlinski, Arnaud Mussot, Rémi Habert, and Thibaut Sylvestre
}

\begin{abstract}
We describe a simple technique that allows for the achievement of widely tunable parametric generation in a photonic crystal fiber. This is achieved by heating the fiber and using a specific phase-matching condition in the normal dispersion regime which is highly sensitive to temperature. Experimental results show a sideband tunability of about $17 \mathrm{THz}$ in the $800 \mathrm{~nm}$ and $1550 \mathrm{~nm}$ spectral bands by heating the fiber from room temperature to $500{ }^{\circ} \mathrm{C}$, leading to a tuning rate of $34 \mathrm{GHz} /{ }^{\circ} \mathrm{C}$. By adding a small tunable continuous-wave seed together with the pulsed pump, we further show the generation of sub-nanosecond pulses tunable around $800 \mathrm{~nm}$ through parametric amplification.
\end{abstract}

Index Terms-Fiber nonlinear optics, four-wave mixing.

\section{INTRODUCTION}

$\mathbf{C}$ OMPACT wavelength-agile short pulsed laser sources are of primary importance for a wide range of applications and for extending the wavelength operation of conventional mode-locked or Q-switched lasers. Fiber optical parametric amplifiers (FOPA) and oscillators (FOPO) appear as promising solutions for realizing such compact devices because of their large wavelength tunability and high conversion efficiency [1]-[5]. For instance, more than $200 \mathrm{~nm}$ and $450 \mathrm{~nm}$ sidebands tunability have been successfully demonstrated in FOPAs and FOPOs, respectively [6], [7]. In most of these devices, the parametric sidebands tunability is in principle obtained by tuning the pump wavelength and thus the group-velocity dispersion (GVD) parameter. However, the GVD parameter can also be tuned by varying the fiber temperature through the thermo-optic effect [8]-[11]. This temperature-induced GVD change has already been studied in parametric amplifiers to either slightly tune FWM sidebands from several nanometers in dispersion-shifted fibers (DSF) [6] or to control the shape of the parametric gain spectrum by longitudinally modulating the fiber temperature [3].

A. Kudlinski, A. Mussot, and R. Habert are with the Université Lille 1, Laboratoire PhLAM-IRCICA, Villeneuve d'Ascq Cedex 59655, France (e-mail: alexandre.kudlinski@univ-lille1.fr; arnaud.mussot@univlille1.fr; remi.habert@phlam.univ-lille1.fr).

T. Sylvestre is with the Université de Franche-Comté, Département d'Optique P.M. Duffieux, Institut FEMTO-ST, Besançon 25000, France (e-mail: thibaut.sylvestre@univ-fcomte.fr).
In this work we demonstrate widely tunable parametric generation simply by heating an optical fiber instead of tuning the pump wavelength. This is achieved by using a photonic crystal fiber (PCF) suitably designed to generate widely spaced parametric sidebands with a specific phase matching condition which is highly sensitive to temperature. By pumping at $1064.5 \mathrm{~nm}$, our experimental measurements show the possibility of tuning the parametric sidebands by almost $17 \mathrm{THz}$ in the $800 \mathrm{~nm}$ and $1550 \mathrm{~nm}$ spectral regions by heating the PCF from room temperature to $500{ }^{\circ} \mathrm{C}$. We also investigate parametric amplification by adding a continuouswave $(\mathrm{CW})$ tunable seed in the $1550 \mathrm{~nm}$ band together with the pulsed pump and show the generation of tunable subnanosecond clean pulses around $800 \mathrm{~nm}$, in a way similar as in Refs. [4], [12].

\section{Temperature Dependence of Four-Wave Mixing}

We consider as in Refs [6], [13], [14] a particular degenerate FWM process occurring in the normal dispersion regime and involving a negative fourth-order dispersion coefficient to be phase-matched. This parametric process generates narrow gain bands widely spaced from the pump wavelength and is highly sensitive to the fiber dispersion. As an example, more than $450 \mathrm{~nm}$ tunability has been successfully demonstrated in a FOPO by tuning the pump wavelength by only $10 \mathrm{~nm}$ [7]. The phase-matching condition reads as $\beta_{2} \Omega^{2}+\left(\beta_{4} \Omega^{4} / 12\right)+2 \gamma P=$ 0 [1]. where $\beta_{j}$ is the $j^{\text {th }}$-order dispersion coefficient at the pump frequency, $\Omega$ is the frequency detuning of the Stokes (low frequency) and anti-Stokes (high frequency) sidebands from the pump, $\gamma$ is the fiber nonlinear coefficient and $P$ is the peak pump power. In the normal GVD regime $\left(\beta_{2}>0\right)$, this equation requires $\beta_{4}<0$ to compensate for the nonlinear phase shift $2 \gamma P$.

Figure 1 shows the phase-matching curve calculated for a pump power of $300 \mathrm{~W}$ and the corresponding FWM sidebands versus the pump wavelength, for the PCF under test (described below). As it can be seen, a small change in the GVD parameter, or equivalently the pump wavelength with respect to the ZDW, significantly shifts the FWM wavelengths. This can be done by varying the fiber temperature that impacts on the refractive index of $\mathrm{SiO}_{2}$-based glasses [15], [16]. The resulting temperature-induced ZDW variation has been estimated as $0.03 \mathrm{~nm} /{ }^{\circ} \mathrm{C}$ and $0.06 \mathrm{~nm} /{ }^{\circ} \mathrm{C}$ in conventional and highlynonlinear DSFs, respectively [8], [11]. Furthermore, depending 


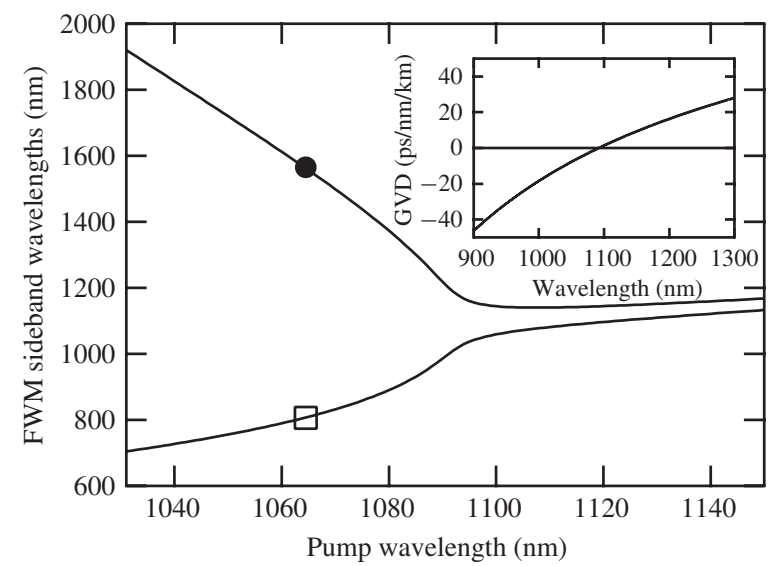

Fig. 1. Phase matching curve of the FWM process under normal dispersion for a pump power of $300 \mathrm{~W}$ (at $20{ }^{\circ} \mathrm{C}$ ), and measurement of the FWM sidebands for a $1064.5 \mathrm{~nm}$ pump (markers). Inset, calculated GVD curve of the PCF under test. The second- and fourth-order dispersion coefficients are, respectively, $\beta_{2}=2.98 \times 10^{-27} \mathrm{~s}^{2} / \mathrm{m}$ and $\beta_{2}=-1 \times 10^{-55} \mathrm{~s}^{4} / \mathrm{m}$ at $1064.5 \mathrm{~nm}$.

on the refractive index profile of the fiber core, the thermal coefficient of GVD variation takes values between -0.004 and $+0.004\left[(\mathrm{ps} / \mathrm{nm} / \mathrm{km}) /{ }^{\circ} \mathrm{C}\right]$ in single-mode fibers [10].

To experimentally study the possibility of tuning FWM sidebands with temperature, we designed and fabricated an air/silica PCF (close to that described in Refs. [17], [18]) with normal GVD at $1064 \mathrm{~nm}$. It has a calculated ZDW of $1093 \mathrm{~nm}$ and a nonlinear coefficient $\gamma$ of $7.2 \mathrm{~W}^{-1} \cdot \mathrm{km}^{-1}$. The simulated GVD curve is displayed in inset of Fig. 1. The group birefringence was also measured to be of about $5 \times 10^{-6}$ at $1.1 \mu \mathrm{m}$. Note that the birefringence can change significantly by varying the fiber temperature and that this effect can also be used to tune the FWM sidebands in a vector FWM configuration, as it has been demonstrated two decades ago [19]. Here we use a scalar FWM process with a phasematching that does not include the fiber birefringence. The PCF was optically pumped with a linearly polarized Nd:YAG laser emitting at a repetition rate of $7 \mathrm{kHz}$ at a wavelength of $1064.5 \mathrm{~nm}$. The pulse duration was measured to be of $0.5 \mathrm{~ns}$. The power launched into the fiber was controlled with a variable attenuator made of a half-wave plate and a polarizer. A short $3 \mathrm{~m}$-long sample of the PCF was used for the parametric generation. The coating was removed all along its length, and it was spliced to two SMF pigtails. The uncoated PCF sample was placed into an oven with the pigtails kept outside. The oven temperature was measured with a type $\mathrm{K}$ thermocouple.

We first recorded the FWM sidebands at room temperature. The measurements are plotted in Fig. 1 as markers and show a very good agreement with the theoretical phase-matching curve. For a $1064.5 \mathrm{~nm}$ pump, the Stokes and anti-Stokes waves are generated at $1565.4 \mathrm{~nm}$ and $806.5 \mathrm{~nm}$ respectively, leading to a large frequency detuning from the pump of 90.1 THz. As mentioned above, heating the fiber leads to a modification of its dispersion properties [8], [10], [11], mainly because of the thermo-optic effect [9]. As a direct consequence, phase-matched FWM sidebands can be continuously tuned by heating the PCF. This has been performed experi-

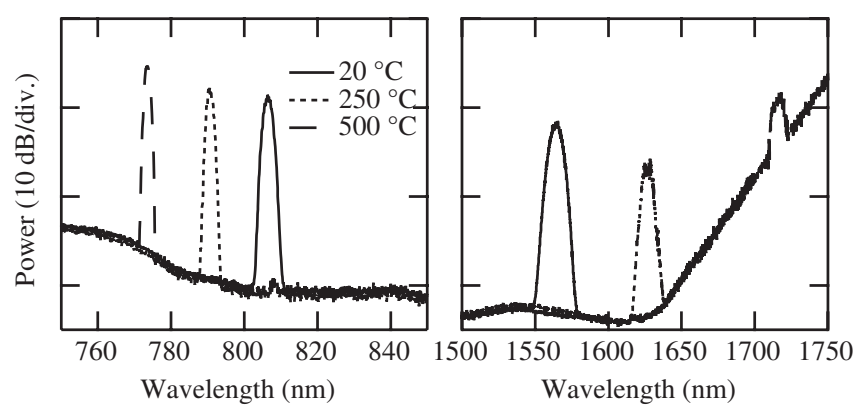

(a)

(b)

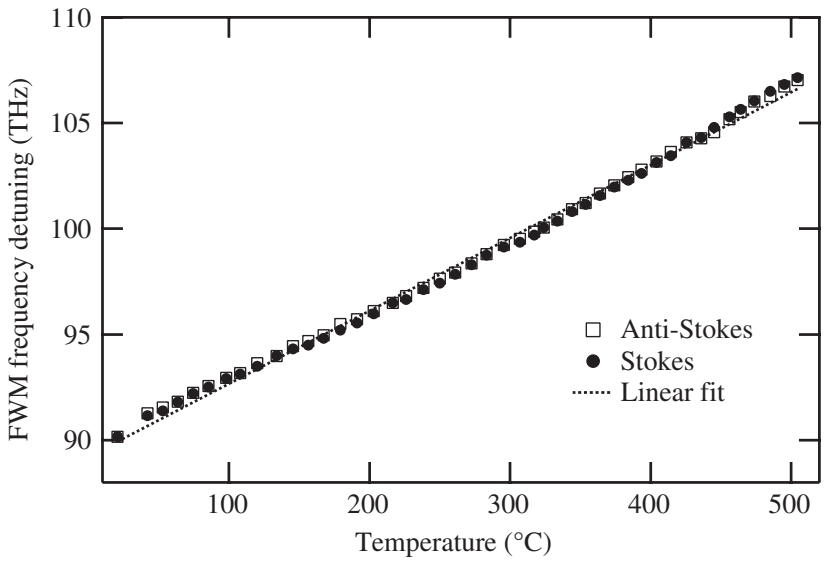

(c)

Fig. 2. (a) and (b) four-wave mixing spectra measured for three PCF temperatures of 20 (solid line), 250 (dotted line), and $500{ }^{\circ} \mathrm{C}$ (dashed line) and for (a) anti-Stokes, (b) Stokes sidebands, and (c) evolution of the FWM sidebands frequency detuning from the pump as a function of temperature. Full circles and open squares correspond, respectively, to anti-Stokes and Stokes waves. The dotted curve is a linear fit.

mentally by sequentially increasing the oven temperature from $20{ }^{\circ} \mathrm{C}$ up to $500{ }^{\circ} \mathrm{C}$, and measuring the $\mathrm{FWM}$ spectrum for each temperature after a stabilization phase of 15 minutes. Figure 2(a) and (b) show typical recorded FWM spectra at 20, 250 and $500{ }^{\circ} \mathrm{C}$, respectively, in solid, dotted and dashed lines. We can easily appreciate the FWM sidebands tunability due to the temperature variations. From $20{ }^{\circ} \mathrm{C}$ to $500{ }^{\circ} \mathrm{C}$, the Stokes and anti-Stokes sidebands are linearly shifted from $1565.4 \mathrm{~nm}$ to $1714.3 \mathrm{~nm}$ and $806.4 \mathrm{~nm}$ to $773.5 \mathrm{~nm}$, respectively. This corresponds to a sidebands tunability of about $17 \mathrm{THz}$ in frequency and a wavelength band of $149 \mathrm{~nm}$ for the Stokes wave and of $33 \mathrm{~nm}$ for the anti-Stokes one. Note that, in Fig. 2(b), the strong increase of the background noise beyond $1650 \mathrm{~nm}$ is due to the low response of the spectrometer in this band and cannot be considered as noise on the FWM sideband. The conversion efficiency of the parametric generation was estimated to be $28 \%$ in the anti-Stokes sideband and $8 \%$ in the Stokes one, which is comparable to measurements reported in Ref. [20]. The lower conversion efficiency to the Stokes wave can be attributed to confinement losses that increase beyond $1550 \mathrm{~nm}$ for this particular PCF design.

Figure 2(c) summarizes the evolution of the Stokes (open squares) and anti-Stokes (full circles) frequency detuning from the pump as a function of fiber temperature. They both closely follow the same linear evolution which confirms the symmetry of the FWM spectrum. A linear fit (dotted line) indicates an 


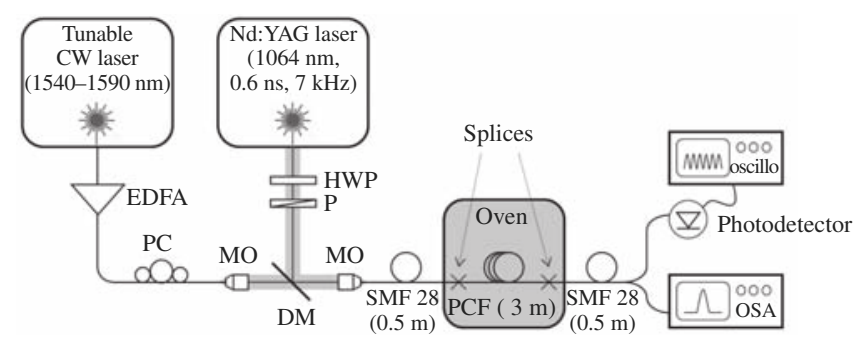

Fig. 3. Experimental setup used for parametric amplification and pulse generation. PC: polarization controller, MO: microscope objective, DM: dichroïc mirror, HWP: half-wave plate, $\mathrm{P}$ : polarizer.

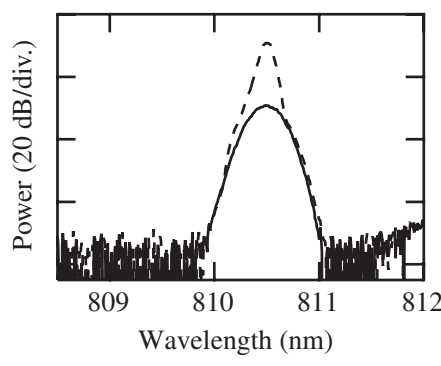

(a)

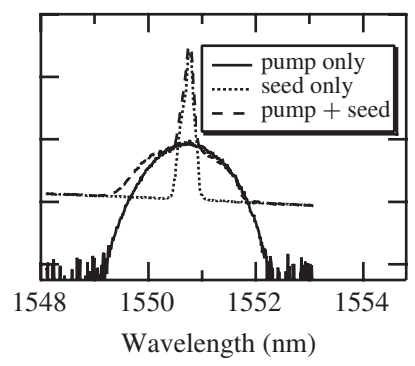

(b)
Fig. 4. Example of output spectra around the (a) anti-Stokes and (b) Stokes sidebands obtained at room temperature with the pump only (solid lines), with the CW seed only (dotted line) and with both the pump and the CW seed (dashed line).

average frequency detuning rate of about $34 \mathrm{GHz} /{ }^{\circ} \mathrm{C}$. From the phase-matching condition, it is possible to determine the ZDW with the method presented in Ref. [21], and thus to deduce its temperature-dependence. In the present case, we found a ZDW variation of $30-60 \mathrm{pm} /{ }^{\circ} \mathrm{C}$, depending on the temperature range, as detailed in Ref. [22]. This is comparable to previous results reported for conventional single-mode fibers [8], [23].

\section{Tunable Pulse Generation Through PARAMETRIC AMPLIFICATION}

In the previous experiment, there was no seed and the parametric sidebands were generated from noise. They usually exhibit a noisy temporal pulse profile and large pulse-topulse power fluctuations due to the high sensitivity of the parametric process to noise [24]. Fortunately, this instability can be significantly reduced by adding a small seed to one of the two FWM sidebands together with the pump pulse. The system thus operates as a parametric amplifier and allows the generation of clean pulses at the anti-Stokes band, as previously demonstrated in Refs. [4], [12] for pulse train generation around $1550 \mathrm{~nm}$.

Here, we carry out a new experiment by using a small tunable $\mathrm{CW}$ probe at the $1550 \mathrm{~nm}$ band and the parametric process delivers clean sub-nanosecond pulses whose wavelength can be tuned around $800 \mathrm{~nm}$ by heating the fiber. The setup of this second experiment is depicted in Fig. 3. The gain medium is an uncoated 3 m-long PCF with a ZDW at $1092 \mathrm{~nm}$, spliced to two SMF pigtails. The ZDW of the PCF used is slightly downshifted as compared to that used in the first experiment, which results in the generation of different FWM sidebands. The seed we used is a CW laser tunable from 1540 to $1590 \mathrm{~nm}$ amplified by an erbium-doped fiber amplifier (EDFA). Both

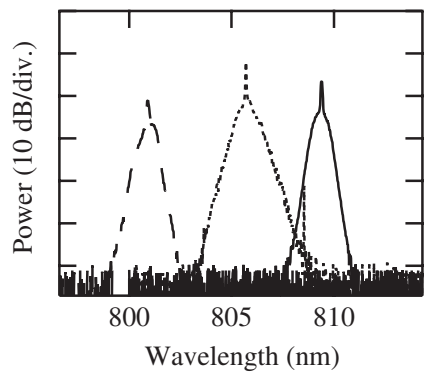

(a)

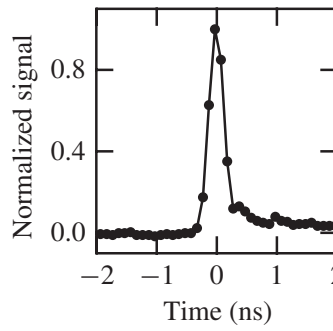

(c1)

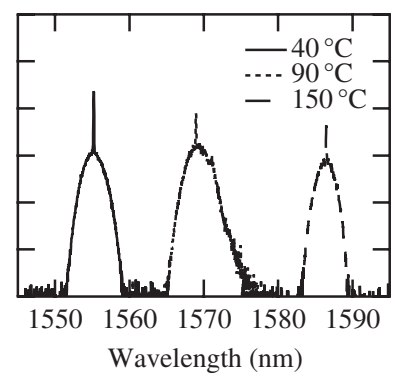

(b)

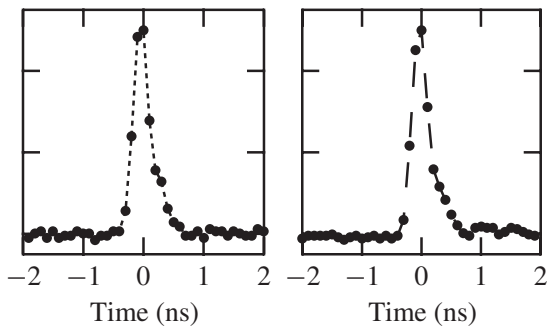

(c2)
Fig. 5. (a) and (b) FWM spectra measured for three temperatures of $40{ }^{\circ} \mathrm{C}$ (solid line), $90{ }^{\circ} \mathrm{C}$ (dotted line) and $150{ }^{\circ} \mathrm{C}$ (dashed line) with both the pump and the tunable $\mathrm{CW}$ seed on. (a) and (b), respectively, show idler generation and parametric amplification of the CW signal. (c1)-(c3) measured temporal trace of filtered pulses corresponding to spectra of Fig. 5(a) (same temperature style code).

the pulsed pump at $1064.5 \mathrm{~nm}$ and the $\mathrm{CW}$ seed fields are combined with a dichroïc mirror and launched into the PCF. They are both linearly polarized and aligned to each other by use of a polarizer for the pump and a polarization controller for the $\mathrm{CW}$ seed. Both input polarization directions were aligned to a neutral axis of the PCF.

Solid lines in Fig. 4 show the output spectra of the antiStokes sideband generated at $810.5 \mathrm{~nm}$ [Fig. 4(a)] and the Stokes one at $1550.8 \mathrm{~nm}$ [Fig. 4(b)] for a pump peak power of about $400 \mathrm{~W}$. The dotted line in Fig. 4(b) corresponds to the injection of $\mathrm{CW}$ seed alone (with the pump off) with an average power of $50 \mu \mathrm{W}$ at $1550.8 \mathrm{~nm}$. Spectra obtained with both the pump and the seed launched into the fiber are depicted in dashed lines.

They show parametric amplification of the seed at $1550.8 \mathrm{~nm}$ and parametric wavelength conversion at $810.5 \mathrm{~nm}$. The parametric gain of the $\mathrm{CW}$ probe was tricky to determine from these spectral measurements because of the low duty cycle $(\mathrm{kHz}$ range) of the pulsed pump and long integration time of our spectrometer. However, these results clearly demonstrate the possibility of parametric amplification with very large detuning from the pump (about $90 \mathrm{THz}$ ). In our configuration in which a $\mathrm{CW}$ seed is amplified with a pulsed pump, the parametric amplification process results in the generation of a pulsed idler around $810 \mathrm{~nm}$. By combining the FWM sensitivity to temperature and pulse generation from parametric amplification, it is thus possible to generate pulses that are wavelength-tunable around $800 \mathrm{~nm}$ by heating the fiber and simultaneously tuning the $\mathrm{CW}$ seed wavelength. To do so, the oven in which the PCF was placed was progressively heated from room temperature $\left(20^{\circ} \mathrm{C}\right)$ to $150{ }^{\circ} \mathrm{C}$. As demonstrated in the previous section, this results to a frequency 
downshift the Stokes wave and a simultaneous frequency upshift of the anti-Stokes one.

Figure 5 shows examples of output spectra measured in such conditions, for temperatures of $40{ }^{\circ} \mathrm{C}$ (solid line), $90{ }^{\circ} \mathrm{C}$ (dotted line) and $150{ }^{\circ} \mathrm{C}$ (dashed line). The Stokes wavelength increases from $1550.8 \mathrm{~nm}$ to $1586.5 \mathrm{~nm}$ from room temperature to $150{ }^{\circ} \mathrm{C}$. For each temperature (every $10{ }^{\circ} \mathrm{C}$ ), the wavelength of the $\mathrm{CW}$ seed was tuned so that it falls at the maximum Stokes parametric gain [see Fig. 5(b)]. Note that we limited our experimental study to $150{ }^{\circ} \mathrm{C}$ because the source we used for our CW seed cannot be tuned to higher than the corresponding Stokes wavelength $(1586.5 \mathrm{~nm})$. Figures 5(c1) to (c3) shows examples of pulse traces around $800 \mathrm{~nm}$ for the three temperatures. They have been recorded with a $10 \mathrm{GHz}$ photo-detector placed after a $850 \mathrm{~nm}$ short-pass filter, and visualized on a $13 \mathrm{GHz}$ analog oscilloscope. The relatively low detection bandwidth with respect to the pulse duration makes it tricky to accurately measure the pulse duration, but the temporal profile looks clean and quasi-Gaussian. These results however provide a proof of principle of the concept of tunable pulse train generation through parametric amplification with very large detuning (of about $90 \mathrm{THz}$ ) from the pump.

\section{CONCLUSION}

We have studied the temperature dependence of the FWM process obtained from a fourth-order phase-matching in a normally-dispersive PCF and demonstrated widely tunable parametric generation detuned by about $100 \mathrm{THz}$ from the pump wavelength of $1064 \mathrm{~nm}$. Our experimental results have shown a significant sidebands tunability of about $17 \mathrm{THz}$ of the parametric sidebands through a temperature increase from $20{ }^{\circ} \mathrm{C}$ to $500{ }^{\circ} \mathrm{C}$. We also investigated parametric amplification by adding a tunable $\mathrm{CW}$ seed with the pulsed pump and showed the generation of clean sub-nanosecond pulses tunable around $800 \mathrm{~nm}$ by changing the PCF temperature.

In addition to widely tunable laser sources, our technique could also find potential application to optical fiber sensors given the ultra-high sensitivity of the sidebands detuning to temperature $\left(34 \mathrm{GHz} /{ }^{\circ} \mathrm{C}\right)$ and the simplicity of the experimental setup based on a microchip laser, a suitable fiber and an optical spectrometer. Moreover, the setup could be made relatively compact by using an appropriate oven such as the one described in Ref. [25].

\section{ACKNOWLEDGMENT}

The authors would like to acknowledge K. Delplace for assistance in fiber fabrication.

\section{REFERENCES}

[1] M. Marhic, Fiber optical parametric amplifiers, oscillators and related devices. Cambridge, U.K.: Cambridge Univ. Press, 2008.

[2] J. E. Sharping, "Microstructure fiber based optical parametric oscillators," J. Lightw. Technol., vol. 26, no. 14, pp. 2184-2191, 2008.

[3] K. K. Y. Wong, M. Marhic, and L. Kazovsky, "Temperature control of the gain spectrum of fiber optical parametric amplifiers," Opt. Express, vol. 13, no. 12, pp. 4666-4673, 2005.

[4] Y. Zhou, K. K. Y. Cheung, S. Yang, P. C. Chui, and K. K. Y. Wong, "Widely tunable picosecond optical parametric oscillator using highly nonlinear fiber," Opt. Lett., vol. 34, no. 7, pp. 989-991, 2009.
[5] T. Sylvestre, A. Kudlinski, A. Mussot, J. F. Gleyze, A. Jolly, and H. Maillotte, "Parametric amplification and wavelength conversion in the 1040-1090 nm band by use of a photonic crystal fiber," Appl. Phys. Lett., vol. 94, no. 11, pp. 111104-1-111104-3, 2009.

[6] M. E. Marhic, K. K. Y. Wong, and L. G. Kazovsky, "Wide-band tuning of the gain spectra of one-pump fiber optical parametric amplifiers," IEEE J. Sel. Topics Quantum Electron., vol. 10, no. 5, pp. 1133-1141, Sep.-Oct. 2004.

[7] G. K. L. Wong, S. G. Murdoch, R. Leonhardt, J. D. Harvey, and V. Marie, "High-conversion-efficiency widely-tunable all-fiber optical parametric," Opt. Express, vol. 15, no. 6, pp. 2947-2952, 2007.

[8] K. Byron, M. A. Bedgood, A. Finney, C. McGauran, S. Savory, and I. Watson, "Shifts in zero dispersion wavelength due to pressure, temperature and strain in dispersion shifted singlemode fibers," Electron. Lett., vol. 28, no. 18, pp. 1712-1714, Aug. 1992.

[9] V. Dangui, H. Kim, M. Digonnet, and G. Kino, "Phase sensitivity to temperature of the fundamental mode in air-guiding photonicbandgap fibers," Opt. Express, vol. 13, no. 18, pp. 6669-6684, 2005.

[10] T. Kato, Y. Koyano, and M. Nishimura, "Temperature dependence of chromatic dispersion in various types of optical fiber," Opt. Lett., vol. 25, no. 16, pp. 1156-1158, Aug. 2000.

[11] J. Hansryd, F. Dross, M. Westlund, A. P. Andrekson, and S. Knudsen, "Increase of the SBS threshold in a short highly nonlinear fiber by applying a temperature distribution," J. Lightw. Technol., vol. 19, no. 11, pp. 1691-1697, Nov. 2001.

[12] T. Torounidis, M. Karlsson, and P. Andrekson, "Fiber optical parametric amplifier pulse source: Theory and experiments," J. Lightw. Technol., vol. 23, no. 12, pp. 4067-4073, Dec. 2005.

[13] S. Pitois and G. Millot, "Experimental observation of a new modulational instability spectral window induced by fourth-order dispersion in a normally dispersive single-mode optical fiber," Opt. Commun., vol. 226, no. 1-6, pp. 415-422, Oct. 2003.

[14] J. Harvey, R. Leonhardt, S. Coen, G. Wong, J. Knight, W. J. Wadsworth, and P. Russell, "Scalar modulation instability in the normal dispersion regime by use of a photonic crystal fiber," Opt. Lett., vol. 28, no. 22, pp. 2225-2227, 2003.

[15] J. H. Wray and J. T. Neu, "Refractive index of several glasses as a function of wavelength and temperature," J. Opt. Soc. Amer, vol. 59, no. 6, pp. 774-776, 1969.

[16] R. Waxler and G. Cleek, "Refractive indices of fused silica at low temperatures," J. Res. Nat. Bureau Stand. Sec. A-Phys. Chem., vol. 75, no. 4, pp. 279-281, 1971.

[17] A. Kudlinski, V. Pureur, G. Bouwmans, and A. Mussot, "Experimental investigation of combined four-wave mixing and Raman effect in the normal dispersion regime of a photonic crystal fiber," Opt. Lett., vol. 33, no. 21 , pp. $2488-2490,2008$.

[18] J. A. Slater, J.-S. Corbeil, S. Virally, F. Bussieres, A. Kudlinski, G. Bouwmans, S. Lacroix, N. Godbout, and W. Tittel, "Microstructured fiber source of photon pairs at widely separated wavelengths," Opt. Lett., vol. 35, no. 4, pp. 499-501, 2010.

[19] M. Ohashi, K. Kitayama, N. Shibata, and S. Seikai, "Frequency tuning of a Stokes wave for stimulated four-photon mixing by temperatureinduced birefringence change," Opt. Lett., vol. 10, no. 2, pp. 77-79, 1985.

[20] W. Wadsworth, N. Joly, J. Knight, T. Birks, F. Biancalana, and P. Russell, "Supercontinuum and four-wave mixing with Q-switched pulses in endlessly single-mode photonic crystal fibres," Opt. Express, vol. 12, no. 2, pp. 299-309, 2004.

[21] M. Droques, B. Barviau, A. Kudlinski, G. Bouwmans, and A. Mussot, "Simple method for measuring the zero-dispersion wavelength in optical fibers," IEEE Photon. Technlo. Lett., vol. 23, no. 10, pp. 609-611, May 2011.

[22] A. Kudlinski, R. Habert, M. Droques, G. Beck, L. Bigot, and A. Mussot, "Temperature dependence of the zero dispersion wavelength in a photonic crystal fiber," IEEE Photon. Technol. Lett., 2011, to be published.

[23] S. H. Kim, D. H. Kim, J. C. Jo, and K. M. Cho, "Temperature dependence of the zero-dispersion wavelength of dispersionshifted fiber," Jpn. J. Appl. Phys., vol. 37, no. 2, pp. L1043-L1045, 1998.

[24] K. Hammani, C. Finot, B. Kibler, and G. Millot, "Soliton generation and rogue-wave-like behavior through fourth-order scalar modulation instability," IEEE J. Photon., vol. 1, no. 3, pp. 205-212, Sep. 2009.

[25] M. Fokine, "High temperature miniature oven with low thermal gradient for processing fiber Bragg gratings," Rev. Sci. Instrum., vol. 72, no. 8, pp. 3458-3461, Aug. 2001. 